\title{
Future Planetary Instrument Capabilities Made Possible by Micro- and Nanotechnology
}

\author{
Stephanie A. Getty*a, Lucy F. Lim*a, Andrej Grubisic ${ }^{\mathrm{a}}$, Adrian E. Southard ${ }^{\mathrm{b}}$, Jerome Ferrance ${ }^{\mathrm{c}}$, \\ Manuel A. Balvin ${ }^{\mathrm{d}}$, Xiang Li ${ }^{\mathrm{e}}$, Timothy Cornish ${ }^{\mathrm{f}}$, Jamie Elsila ${ }^{\mathrm{a}}$, Larry Hess ${ }^{\mathrm{d}}$, Carl Kotecki ${ }^{\mathrm{d}}$, John \\ Hagopian $^{\mathrm{g}}$, William B. Brinckerhoff ${ }^{\mathrm{a}}$
}
aSolar System Exploration Division, NASA Goddard Space Flight Center, Greenbelt, MD 20771; ${ }^{b}$ Universities Space Research Association, Columbia, MD 21046; ' J2F Engineering, Inc, Charlottesville, VA; ${ }^{\mathrm{d} D e t e c t o r}$ Systems Branch, NASA Goddard Space Flight Center, Greenbelt, MD 20771; ${ }^{\mathrm{e} U n i v e r s i t y ~ o f ~ M a r y l a n d ~ B a l t i m o r e ~ C o u n t y, ~ B a l t i m o r e, ~ M D ~} 21250 ;{ }^{\mathrm{f}}$ Zeteo Tech LLC, Sykesville, MD 21784; ' ${ }^{\mathrm{L}}$ ambda Consulting, Harwood, MD 20776

\begin{abstract}
A number of new instrument capabilities are currently in maturation for future in situ use on planetary science missions. Moving beyond the impressive in situ instrumentation already operating in planetary environments beyond Earth will enable the next step in scientific discovery. The approach for developing beyond current instrumentation requires a careful assessment of science-driven capability advancement. To this end, two examples of instrument technology development efforts that are leading to new and important analytical capabilities for in situ planetary science will be discussed: (1) an instrument prototype enabling the interface between liquid separation techniques and laser desorption/ionization mass spectrometry and (2) an addressable excitation source enabling miniaturized electron probe microanalysis for elemental mapping of light and heavy elements.
\end{abstract}

Keywords: planetary, instrumentation, analytical, in situ, mass spectrometer

*stephanie.a.getty@nasa.gov; phone 1301 614-5442; lucy.f.lim@nasa.gov; phone 1301 286-1546; fax 1301 555-8760; science.gsfc.nasa.gov

\section{INTRODUCTION}

The past decade has seen a revolution in our understanding of the range of possible chemical processes in planetary environments throughout our solar system. From the discovery that indigenous organics on Mars could encompass a range of complexities [1,2], to the discovery and beginnings of exploration of salty water plumes originating from a buried ocean on Enceladus [3], and including the exciting realization following recent exploration of Europa, Titan, and Ceres that (beyond Enceladus) ocean worlds are a class of objects harboring new insights into what constitutes habitability and prebiotic chemistry [4].

These revelations and the scientific investigations that will follow them rely fundamentally on in situ mission payloads that are capable, robust, rugged, reliable, and compact. Multiple factors therefore contribute to the optimized choice of scientific payload, but instrument mass and volume are consistently constrained for most mission concepts. Here we describe two recent instrument development efforts that leverage miniaturized components to achieve science return beyond the current state-of-the-art for flight instruments of their kind. Both would be compelling and complementary elements of a future science payload focusing on chemistry and composition of planetary surfaces, as the community considers the next steps to exploring our solar system [5-7].

\subsection{Organic Analysis for Planetary Missions}

Organics are critical to our understanding of how life took root on Earth - and for our future ambitions to understand whether life existed or exists in other habitable environments in our solar system. As context for the emergence of biochemistry, therefore, it is necessary to understand the complexity and diversity of organic chemistry that is possible under abiotic conditions. The rich study of meteorites that have been delivered to Earth, coupled with remote and in situ 


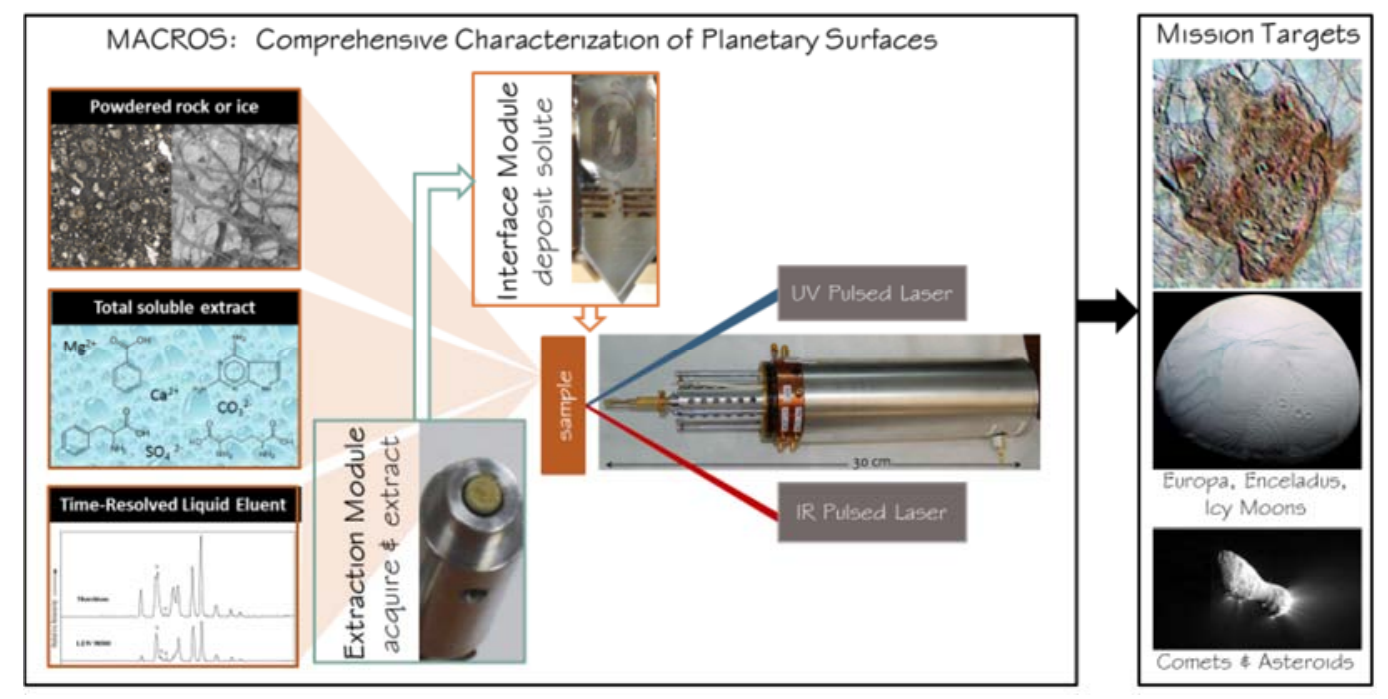

Figure 1: The MACROS instrument enables direct, sensitive analysis of liquid-phase processed samples by laser desorption/ionization mass spectrometry.

exploration of comets and carbonaceous asteroids, such as the Rosetta orbiter $[8,9]$ and the OSIRIS-REx sample return mission now at Bennu [10], are deepening our understanding of the emergence of prebiotic chemistry that would have plausibly provided feedstock to emerging biology on Earth and potentially elsewhere. As those organics mature under various regimes in chemical conditions, the range of possible organic structures evolves, with the end member in an environment where life evolves being a biochemically constrained subset of all possible chemistries that is a signature of life.

Mars was once a warmer, more watery environment that could have hosted biology in its past, with the potential remaining for harboring present-day life in regions of particular "special interest." Since 2012, the Curiosity rover has been exploring an ancient lake bed at Gale Crater in the mid-latitudes of Mars. With its sophisticated chemical laboratory, the Sample Analysis at Mars (SAM) instrument suite, Curiosity has discovered small organic fragments and evidence for macromolecular forms of carbon that are indigenous to the planet. These seminal discoveries have been possible even in the presence of mitigating factors, including deleterious levels of cosmic radiation at the surface of Mars, as well as a particularly reactive perchlorate salt, both of which have overprinted the indigenous organic composition. Future Mars instrumentation, therefore, will benefit from these achievements and leverage design and protocol improvements to take the next steps in exploring Mars (e.g., [11]) and other high-priority planetary surfaces.

A promising future direction to complement mass spectrometer investigations into the indigenous organic chemistry of planetary materials would be the capability to process and analyze samples in the liquid phase. This is relevant not only to samples accessible in the liquid phase, as found in places like Europa, Enceladus, and Titan, but also to the prospect of in situ solvent extraction of solid-phase surface materials [12]. Solvent extraction enables tailoring of the extraction chemistry to the expected organic composition of the targeted environment; it also provides the ability to couple liquidphase separations of semi-volatile and polar fractions of the sample with mass spectrometry to provide sensitive and structurally resolved analysis $[13,14]$. Efforts to develop an end-to-end system to couple liquid-phase sample handling and separation techniques with laser desorption time-of-flight mass spectrometry have demonstrated some recent success enabled by miniature, microscale, and nanoscale components, as shown in Figure 1 and described in Section 2 below.

\subsection{Elemental Mapping on Planetary Surfaces}

In the standard laboratory technique of electron probe microanalysis ("EPMA"), an electron beam knocks out inner-shell electrons from atoms in the target material, thus exciting X-ray line emission at energies characteristic of the elemental composition of the target's surface. By miniaturizing the electron source, the Mini-EPMA is designed to conduct an EPMA-like analysis in situ. By combining efficient microscale compositional mapping of major (Na through $\mathrm{Ni}$ ) and minor rock-forming elements with a new light-element (C/N/O/F) capability, the mini-EPMA (Fig. 2) will significantly advance our capability for remote in situ analysis of samples of planetary, asteroidal, and cometary material. Elemental composition provides key evidence about the processes by which rocks, soils, and ices were formed and altered (e.g., 
accretion, differentiation, and hydrothermal alteration), thus recording past stages in solar system evolution.

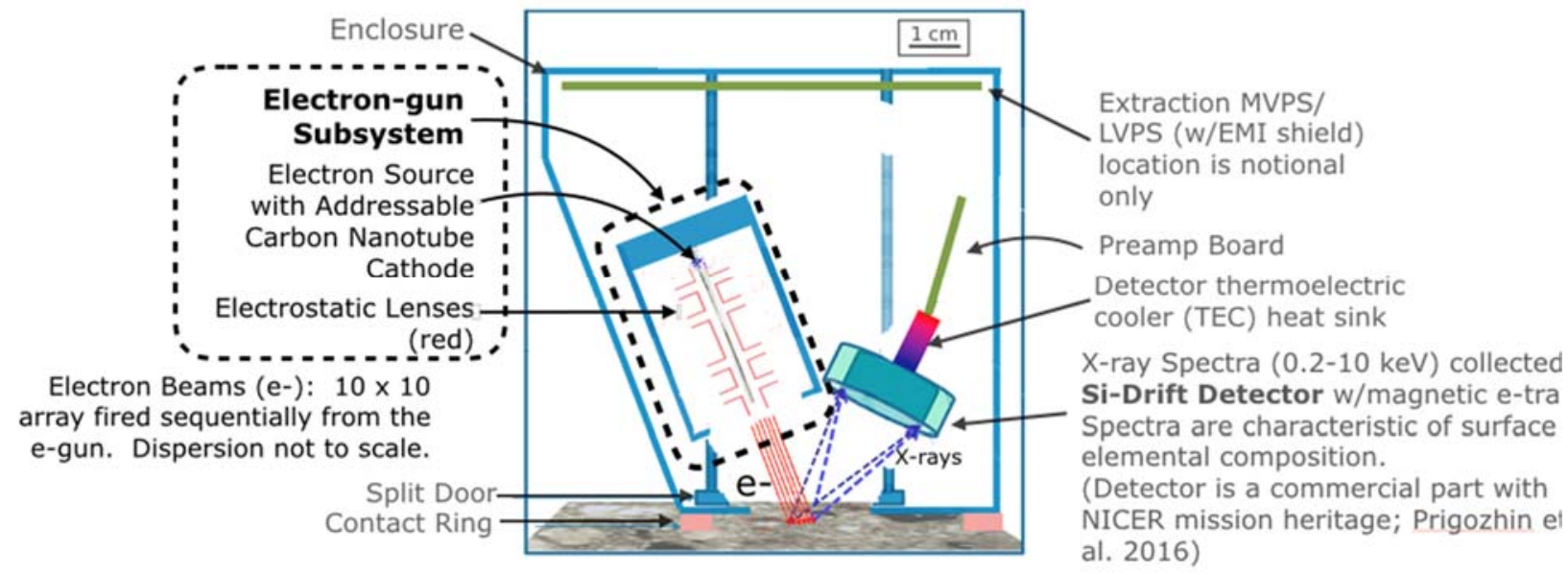

Figure 2: The electron gun will illuminate 100- $\mu \mathrm{m}$ spots on the natural surface, thus enabling in situ mapping of elemental composition on the scale of the beam diameter. The mini-electron probe ("EPMA") flight concept: electrons are drawn out of the addressable-cathode emitter by the cathode/grid extraction voltage, then accelerated by the lens stack into the planetary/asteroidal/cometary surface, exciting characteristic X-ray line emission. The X-ray spectra measured by the Sidrift detector are then analyzed using standard EPMA techniques to give the surface composition of the illuminated spot. In this way, a 10x10 grid of e-beam spots activated in sequence will non-destructively produce a fine-scale map of elemental composition.

Instrumentation like the mini-EPMA, when coupled with analytical instrumentation like a mass spectrometer, can improve analysis of the planetary surface composition through complementary elemental quantification to the organic and mineralogical composition provided by a mass spectrometer instrument.

\section{INSTRUMENT DESIGN AND TECHNOLOGY: MACROS}

The Molecular Analyzer for Complex Refractory Organic-rich Surfaces (MACROS) instrument development has optimized an interface between liquid-phase sample, either ice melt or an extract, with laser desorption/ionization mass spectrometry (LDMS). The analytical power of this coupling includes several benefits: (1) a solvent extraction is a gentle method of mobilizing targeted components from a complex sample, (2) liquid processing is compatible with purification and concentration techniques such as desalting and solid phase extraction, (3) a liquid-phase sample is compatible with chromatographic or electrophoretic separation techniques to complement mass spectrometry, and (4) liquid techniques like liquid chromatography-mass spectrometry have been shown to be orders of magnitude more sensitive than gas chromatography-mass spectrometry, particularly for complex and polar organic molecules. The following description reports successful developments of key elements of an interface between liquid processing and an LDMS prototype.

\subsection{Mass Analyzer}

Current performance testing implements a highly capable, reversible-polarity time-of-flight mass spectrometer (LD-TOFMS) for laboratory demonstrations. The mass analyzer itself measures $\sim 30 \mathrm{~cm}$ long and $5 \mathrm{~cm}$ in diameter, with a demonstrated mass range of 1 to at least 150,000 Da and a typical mass resolution of $m / \Delta m \sim 500$. The ionization process is governed by the interaction of the sample with a focused, pulsed UV laser system (Nitrogen $337 \mathrm{~nm}, \mathrm{Nd}: Y A G 355 \mathrm{~nm}$, or Nd:YAG $266 \mathrm{~nm}$ ) with an effective spot size of 50-100 microns. Both positive and negative ions can be detected separately. The instrument also includes a pulsed pin ion gate, which provides a number of analytical benefits achieved: (1) improved sensitivity by selectively eliminating detector saturation that can be produced by high abundance cation or anion signals appearing at low mass and (2) detection of product ion spectra through fragment analysis [14]. The existing LD-TOF-MS prototype has been demonstrated to operate in low-pressure environments $\left(\mathrm{P}<10^{-4}\right.$ Torr). The TOF-MS is naturally suited to airless bodies because its operation is optimized at vacuum (to maximize mean free path). It should be noted that the MACROS interface components are compatible with multiple types of mass spectrometry and a range of planetary environments. 


\subsection{Liquid Extraction and Separation}

During LDMS analysis of a solid surface of a natural sample, some insoluble materials, such as salts, may dominate the spectra and reduce the detection sensitivity of the targeted organics. Liquid-phase extraction methods to gently isolate the soluble organic and inorganic fractions of a planetary powder is a solution to this problem, which can enhance the detection sensitivity and detailed analysis of these analytes. In addition, careful solvent selection allows the analysis to be tuned to focus on specific, minor species of interest. In MACROS development work, extracts achieved from the powder samples are transferred and deposited through a capillary onto a substrate for LDMS analysis, with enrichment of the sample accomplished by tuning the deposition time to accumulate extract. This simple extraction capability is compatible with a separation step, wherein discrete spot depositions can record fractions exiting a separation column and provide a complementary chromatogram.

\subsection{Coupling a Liquid Sample to LDMS}

The interface between the MACROS fluidic subsystem and the LDMS is characterized by a capillary termination at a flat sample plate; this allows the liquid sample to be deposited onto the sample plate. The sample plate can be prepared in-line or off-line for matrix-assisted laser desorption/ionization (MALDI), as further described in Section 2.4. After liquid deposition, the residue from the deposited sample is then aligned with the mass spectrometer inlet and laser source for LDMS analysis.

The sensitivity of the sample deposition process is under investigation as a function of flow rate, substrate temperature, and ambient pressure, including high vacuum conditions. Early results are reported in [12]; end-to-end system-level testing is ongoing and will be presented in future reports.

\subsection{Inorganic MALDI}

In traditional MALDI-MS, an organic acid (or MALDI "matrix") is mixed with a purified protein or peptide solution for the purpose of promoting ionization of intact large organic molecules during the MS analysis. The organic MALDI matrix, however, would require storage and deposition in a flight implementation. One alternative worth exploring for its relative simplicity is an inorganic MALDI (iMALDI) matrix (e.g., cobalt nano-particles [16]). A suitable iMALDI matrix would reduce contamination concerns and achieve the same desorption/ionization enhancement goal as an organic matrix, but in a cruise-stable formulation that would not add appreciable complexity to the sample preparation requirements of the instrument.

Towards this end, we have studied the characteristics of selected iMALDI matrices for improving MS instrument detection sensitivity and analytical capability. Among these, a thin layer of silicon nanopowder (diameter $<100 \mathrm{~nm}$ ) was deemed an optimal solution for ease of preparation and low background signal in both positive and negative ion modes. A scanning electron micrograph of this iMALDI substrate is shown in Figure 4. We have demonstrated efficacy of this iMALDI matrix in the sensitive detection of a wide range of organics, including aliphatic hydrocarbons, carboxylic acids, nonaromatic amino acids, peptides, and organic polymers. For example, as shown in Figure 3, phthalic acid alone cannot be directly detected by LDI in positive mode, while the presence of a Si nanopowder underlayer clearly promotes the ionization of protonated species, providing over two orders of magnitude increase in intensity of the signal (referenced to the noise level). In addition to the sensitivity enhancement, the nano-particles surface is a more effective absorption base for the sample, so that the deposition efficiency from liquid or extract outlet onto the substrate can be increased [12]. This surface preparation method could be preapplied during instrument development, poses minimal risk of forward contamination, is expected to be immune to degradation by radiation and temperature cycling, and would be stable during a long-duration cruise phase. 


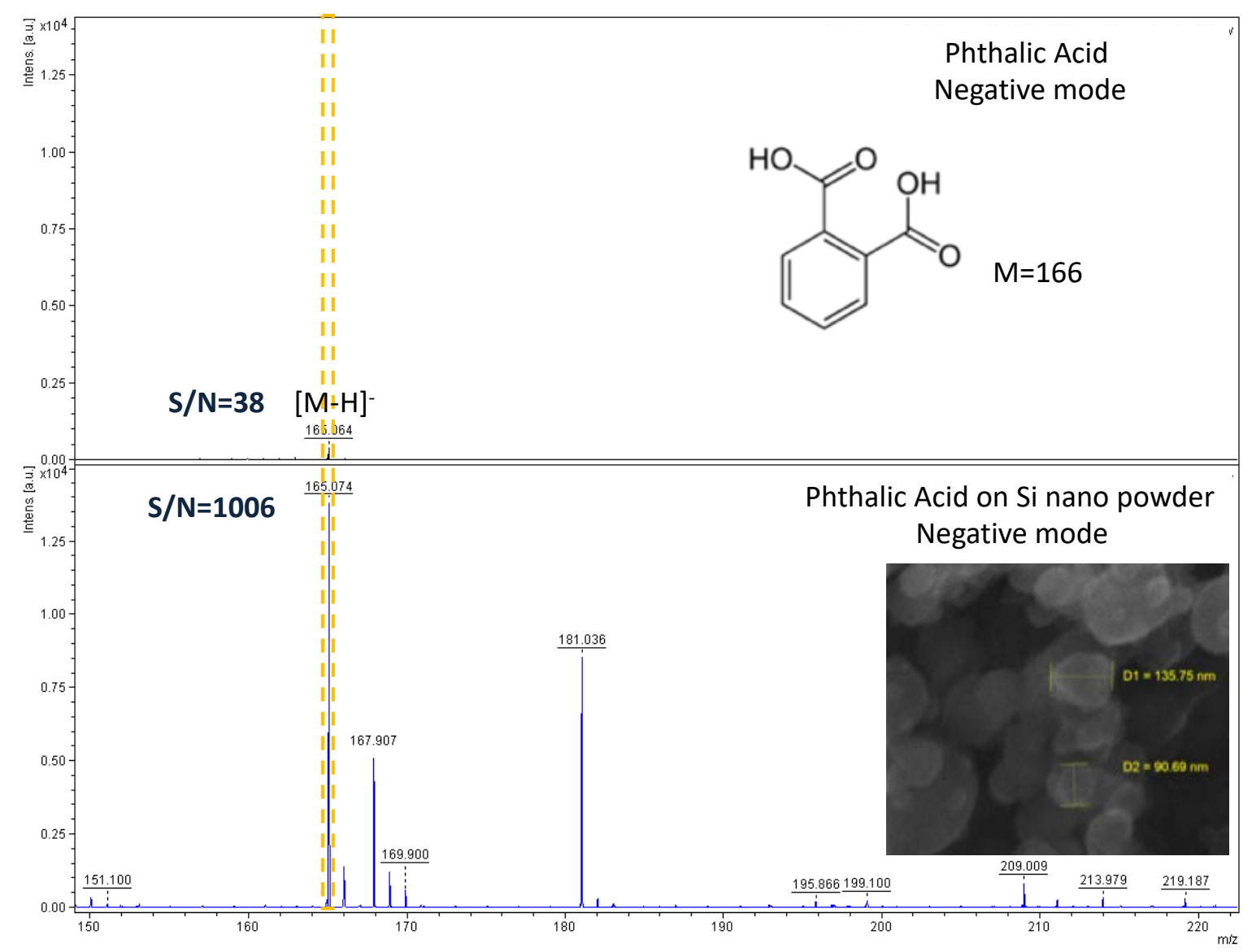

Figure 3: The use of silicon nanopowder amplifies the desorption and ionization efficiency of a relevant planetary analog standard, phthalic acid, deposited in solution-phase and subsequently analyzed in LDMS.

\section{INSTRUMENT DESIGN AND TECHNOLOGY: MINI ELECTRON PROBE MICROANALYSIS}

We have been developing an addressable-array carbon nanotube ("CNT")-based electron source that can be applied to make the standard laboratory technique of electron probe microanalysis (EPMA) feasible in situ on a lander mission (Fig. 2). In the EPMA technique, an electron beam knocks out inner-shell electrons from atoms in the target material, thus exciting X-ray line emission at energies characteristic of the elemental composition of the target's surface. The MiniEPMA is designed to provide rapid in situ determination of the major and minor elemental compositions of lunar, planetary, asteroidal, and cometary rocks, soils, and ices, including the light elements C, N, O, and F; and to return 10x10 element compositional maps of planetary materials at $100-\mu \mathrm{m}$-scale spatial resolution, a scale not yet achieved in flight instrumentation.

Fig. 4 illustrates the important compositional heterogeneity and structure accessible at the $100 \mu \mathrm{m}$ scale in three extraterrestrial rocks, showing how fine-scale texture can offer key information about the origin and evolution of planetary materials that is unavailable from bulk compositional measurements. 

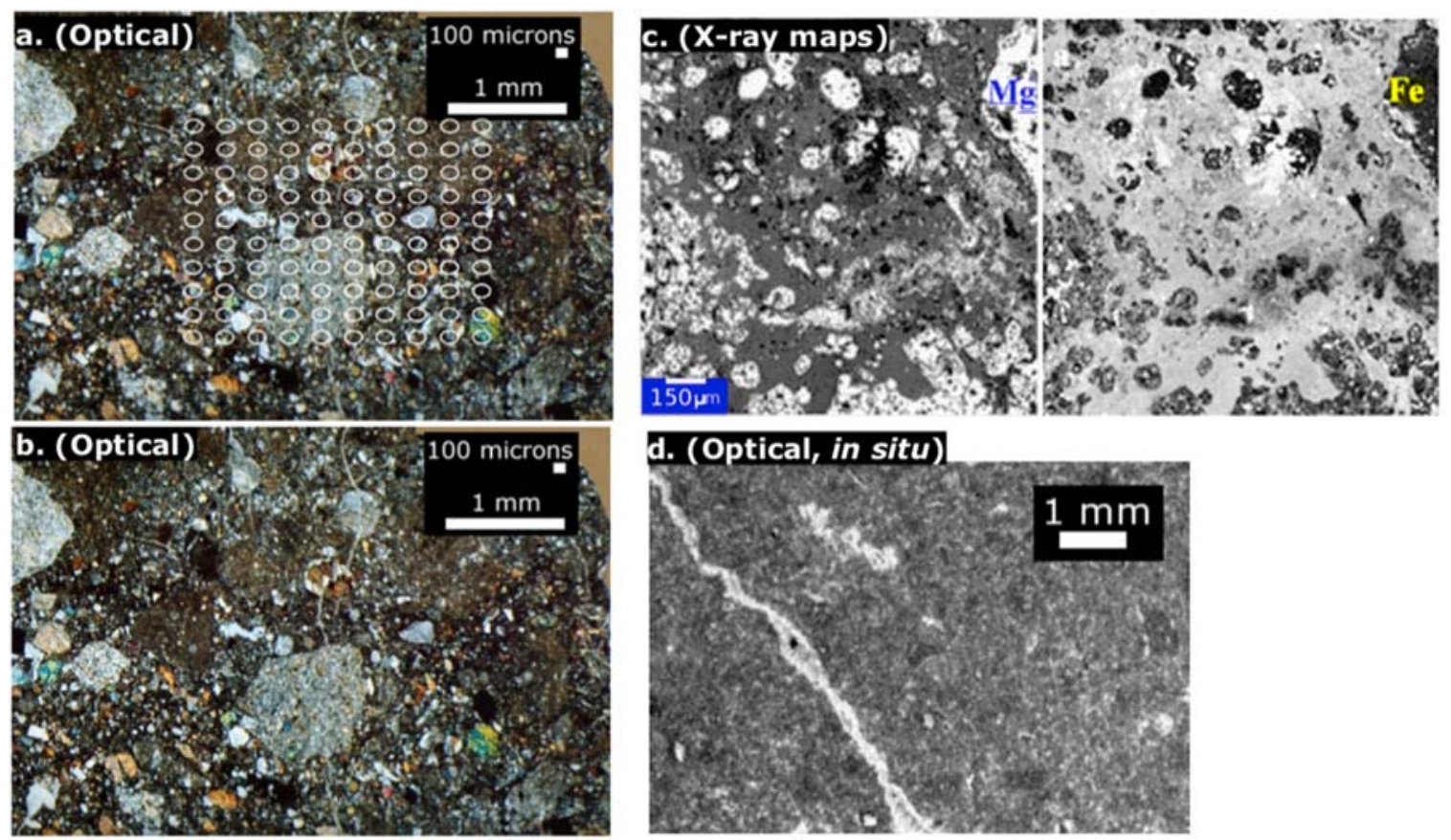

Figure 4: The addressable carbon nanotube (CNT) e-beam source will permit compositional mapping with a spot size of $100 \mu \mathrm{m}$, a scale on which many solar system materials exhibit important structure. (a, b) Lunar breccia DHO 1180 [20] with and without a candidate mini-EPMA spot pattern (based on electron optical modeling) superimposed. (c) CV chondrite Vigarano, USMN 6295-3 in X-ray emission (Mg, Fe) [21]. (d) “Adirondack," basalt, Gusev Crater, Mars.

One important feature of the mini-EPMA is its ability to directly detect characteristic X-rays from carbon, nitrogen and oxygen (Ka 277-525 eV; [17-19]), which represents a significant advance over existing X-ray spectroscopy (XRS) flight instrumentation (Table 1 and [22]). The low-energy detection capability in this compact instrument is enabled by a combination of high detector sensitivity and the use of a polymer or $\mathrm{Si}_{3} \mathrm{~N}_{4}$ window in place of the standard Be windows, which absorb low-energy X-rays (e.g., [23]).

Table 1: Flight Instruments with XRS Capabilities

\begin{tabular}{|c|c|c|c|c|}
\hline \multicolumn{5}{|c|}{$\begin{array}{l}\text { Note that all systems prior to the } 2020 \text { PIXL have had spatial resolutions }>1 \mathrm{~cm} \text {, over } 100 \\
\text { times larger than the } 100 \mu \mathrm{m} \text { spot size of the Mini-EPMA electron beam. PIXL has a } 120 \mu \mathrm{m} \\
\text { spot size (Allwood et al., 2016) }\end{array}$} \\
\hline Instrument & X-ray Detector & Excitation Source & Elements $^{a}$ & Time \\
\hline MER APXS (2004) & $\operatorname{SDD}\left(10 \mathrm{~mm}^{2}\right)$ & $\begin{array}{l}\text { Radioisotopes } \\
(\mathrm{Cm}-244)\end{array}$ & $\geq \mathrm{Na}(\mathrm{Z}=11+)$ & $6-8 \mathrm{~h}$ \\
\hline $\begin{array}{l}\text { MSL CheMin (2011) } \\
\text { XRF/XRD }\end{array}$ & $\mathrm{CCD}$ & $\begin{array}{l}28 \mathrm{keV} \text { Co X-ray } \\
\text { tube }\end{array}$ & $\mathrm{Mg}-\mathrm{Fe}$ & $\begin{array}{l}\text { hours; sample } \\
\text { powdering and } \\
\text { delivery needed }\end{array}$ \\
\hline MSL APXS (2011) & $\operatorname{SDD}\left(10 \mathrm{~mm}^{2}\right)$ & $\begin{array}{l}\text { Radioisotopes } \\
(\mathrm{Cm}-244)\end{array}$ & $\geq \mathrm{Na}(\mathrm{Z}=11+)$ & $3 \mathrm{~h}$ \\
\hline Mars2020 PIXL (2020) & $\operatorname{SDD}(x 2)$ & $\begin{array}{l}\text { Microfocus } \mathrm{X}- \\
\text { ray tube, } 28 \mathrm{kV}\end{array}$ & $\geq \mathrm{Na}(\mathrm{Z}=11+)$ & $<2 \mathrm{~min} /$ point \\
\hline $\begin{array}{l}\text { Mini-EPMA (this } \\
\text { work) }\end{array}$ & $\operatorname{SDD}\left(70 \mathrm{~mm}^{2}\right)$ & $\begin{array}{l}\text { CNT electron } \\
\text { beam }\end{array}$ & $\geq \mathrm{C}(\mathrm{Z}=6+)$ & $\begin{array}{l}1 \mathrm{~min} / \text { point; } \\
2-3 \mathrm{~h} / \mathrm{map}\end{array}$ \\
\hline
\end{tabular}

${ }^{a} \mathrm{Z}=$ atomic number 
This low-energy detection capability enables important science. Detection of carbon in particular, is of interest because in addition to carbon's astrobiological importance, it is structurally important to a class of minerals (carbonates) that are key to understanding aqueous processing in solar system materials. Other forms of carbon (such as graphite, carbides, and organic carbon) are also important constituents of several classes of meteorites, notably ureilites and various carbonaceous chondrites in which carbon can represent up to $5 \%$ by weight of the whole rock.

\subsection{Electron Source}

The addressable carbon nanotube cathode electron beam will permit compositional mapping with a spot size of $100 \mu \mathrm{m}$. By activating the cathode elements in sequence (Fig. 2), it will be possible to assemble concentration maps similar to those illustrated in Fig. 4c and to return compositional analyses of individual mineral grains and rock microstructure. Additionally, the electron beam is nondestructive, allowing other analytical techniques to be employed subsequently on the same sample.
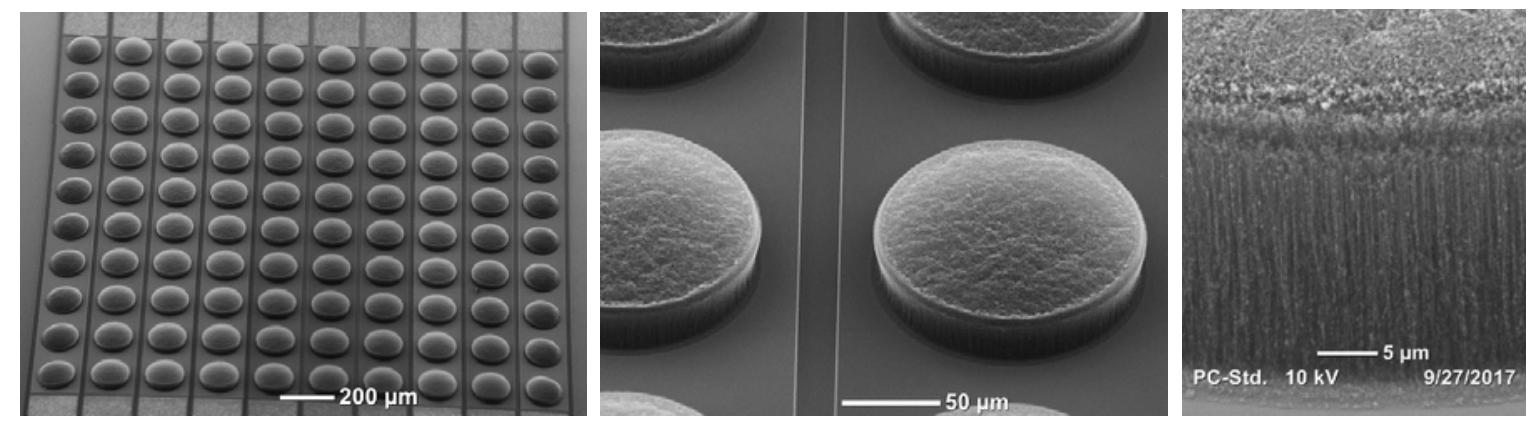

Figure 5: Examples of successful carbon nanotube emitter growth on etched silicon-on-insulator (SOI) wafers. These cathodes show consistent growth, good CNT density within forests, and good adhesion of the CNTs to the substrate.

\subsection{Addressability and Focusing}

The critical new technology enabling the mini-EPMA instrument is the addressable array of field emitters. Each field emitter is a carbon nanotube "forest" grown in a circular $100 \mu \mathrm{m}$ region on a wafer substrate (Fig. 5). An extraction "grid" electrode sits above the emitters; a voltage bias between the cathode and the grid activates the field emission. The key challenge was to achieve robust, consistent nanotube growth on a wafer patterned for electrical addressability via wirebonding and row-wise electrical isolation. Robustness dictates how many hours these devices can emit, while consistency in forest height is necessary to selectively bias a single pixel and inhibit emission from neighboring ones.

The addressable electron source is formed by mounting a cathode chip and a "grid chip", which includes the extraction grid, to the same ceramic fan out board which in turn is supported by a PCB (Figure 6). The cathode chip is formed by etching the silicon device layer of an SOI wafer into rows of silicon strips and depositing growth catalyst in the shape of filled 130 um diameter circles onto these strips (see Figure 4). Then, carbon nanotube growth by chemical vapor deposition is performed. The grid chip is formed by etching strips of silicon with holes in the device layer of a separate SOI wafer. The strips of silicon on the grid chip run orthogonal to the strips on the cathode chip and the holes in these strips are aligned to the carbon nanotube pillars on the cathode chip during the mounting process. To "turn on" a specific field emitter in the array, the voltage at one of the cathode strips is reduced by $100 \mathrm{~V}$ and the voltage at one of the grid strips is increased by $100 \mathrm{~V}$, increasing the voltage bias across the emitter at the intersection point of the two strips by $200 \mathrm{~V}$ and activating field emission. This necessarily introduces an additional bias of $100 \mathrm{~V}$ for emitters along the same cathode strip and grid strip; hence, additional designs that control addressability at the pixel level are being examined; however, this approach requires using a larger pitch to avoid beam broadening due to fields between adjacent emitters. 


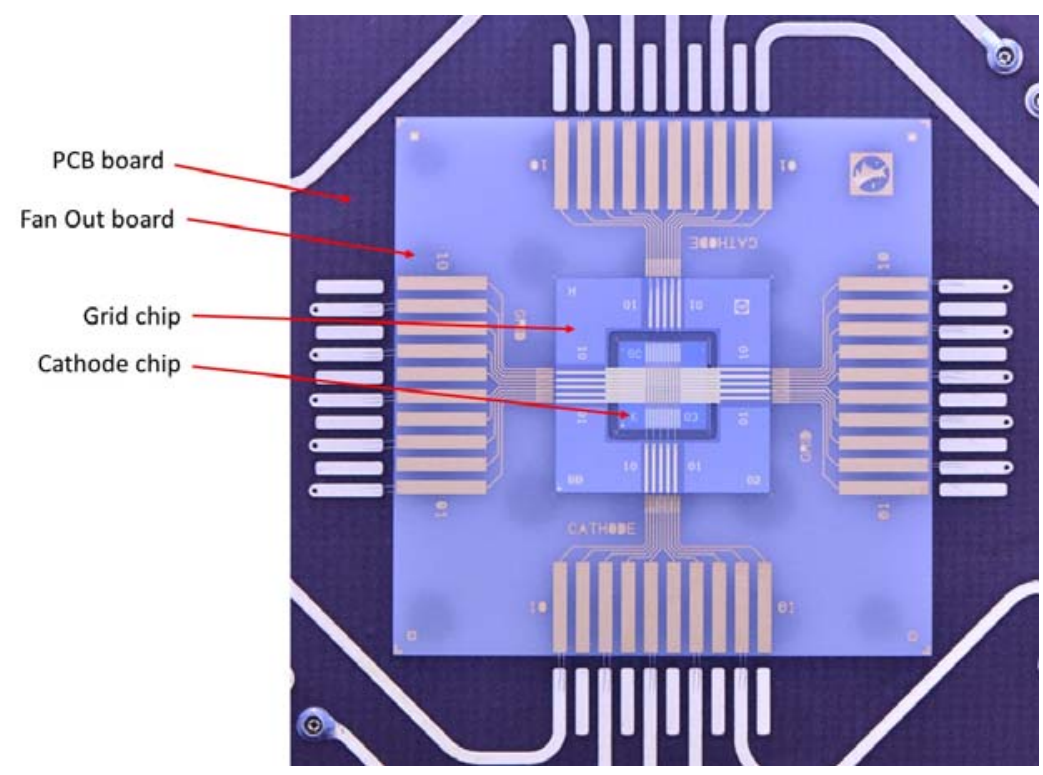

Figure 6: Optical image taken of addressable electron source mounted to a fan out board which is mounted to a PCB board. Wire bonds are used to connect the gold bond pads on the cathode and grid chips, grid chip and fan out board, and the fan out board and PCB board.

Focusing of the beam emitted by each emitter is accomplished using a five element lens stack capable of many operating modes such as acting as an afocal lens (Figure 7). As described by [24] the afocal mode requires the second focal point of the first lens (elements 1-3 from left to right) and the first focal point of the second lens (elements 3-5) to coincide.

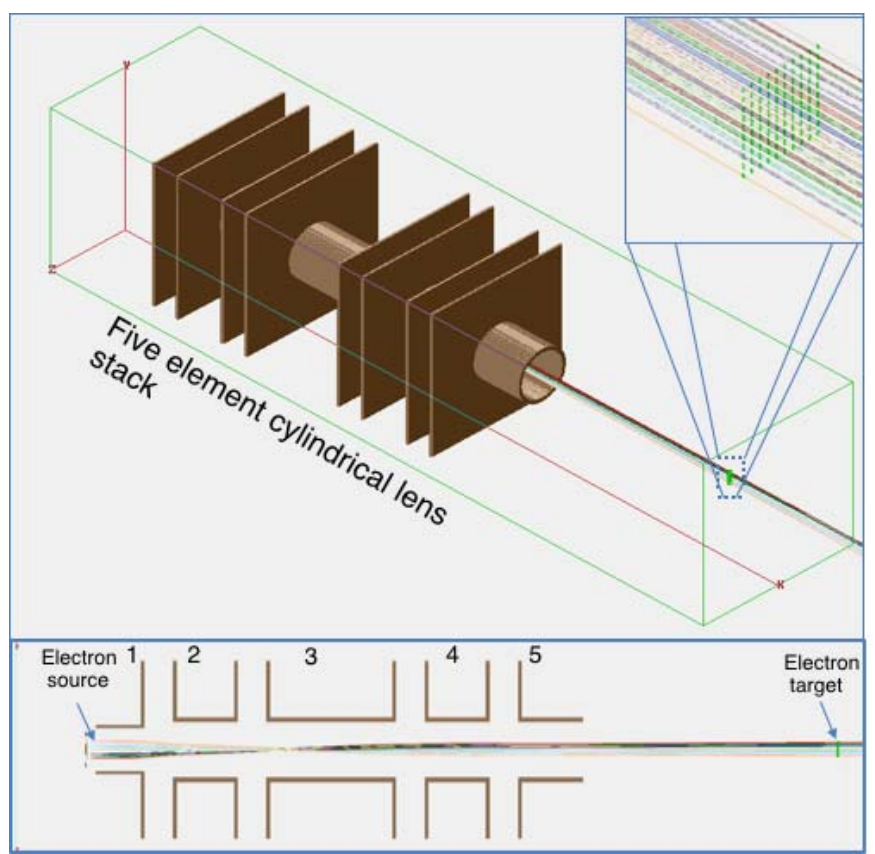

Figure 7: Results of a simulation (SIMION) of electrons emitted from a line source and accelerated to $15 \mathrm{keV}$ before reaching a target $55 \mathrm{~mm}$ from the end of the last lens. (Top) 3D view of lens stack (Top; Inset) Zoom-in view of colored electron trajectories crossing the target plane (intersection points in green). (Bottom) Cross section of simulation showing beam focusing in the middle element of the five element stack where the two focal points must coincide. Lens elements $1-5$ are indicated. 


\subsection{Emitter Testing}

To date, cathode fabrication batch runs have produced satisfactory yields of field emitters that have performed well in whole-chip field emission testing, both in levels of current emitted (from 10 to several hundred $\mu$ A over 100 emitters) and in cathode performance over hundreds of hours of emission (lifetesting; Fig. 8) similar to previous reports for CNT emitters in other applications (e.g., [25]).

The electron source will be tested at the extraction voltage, then assembled together with a high-voltage lens stack. The resulting e-gun subsystem will then be validated at the operational voltage, $-15 \mathrm{kV}$. Systems-level testing is currently ongoing and will be reported separately.

(a)

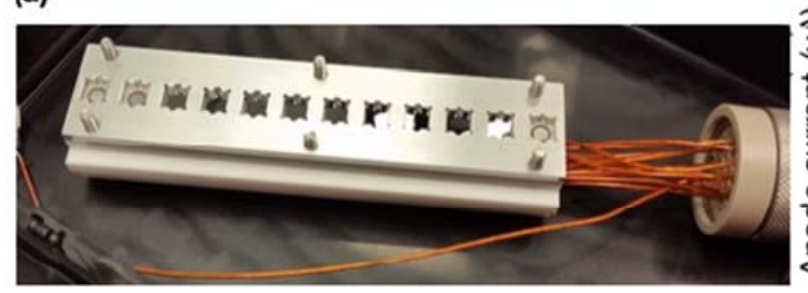

(b)

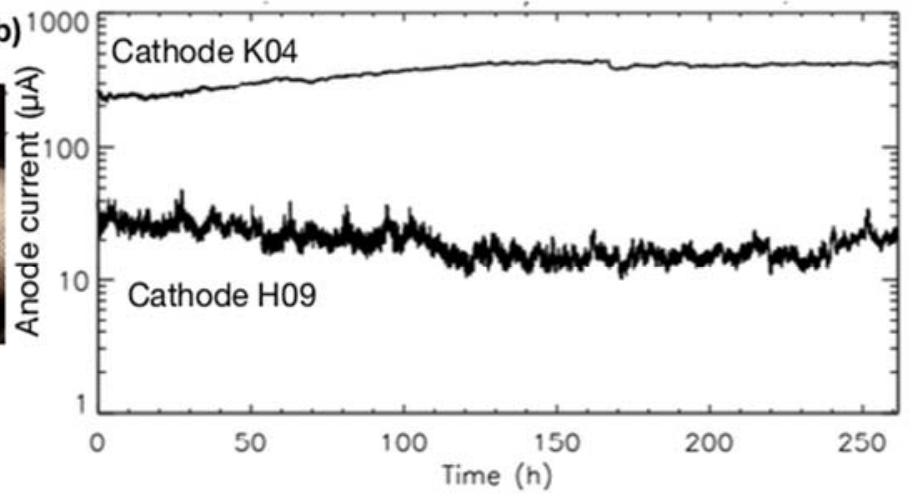

Figure 8: Cathode whole-chip testing validated levels of current emission and longevity of performance. a. Test fixture for whole-chip cathode activation and current measurement. Twelve cathodes at a time can be loaded. A flat anode takes the place of the extraction grid in Fig. 6 so that a constant voltage bias is applied across the whole chip under high vacuum $\left(<10^{6}\right.$ Torr $)$. Current emitted by the whole CNT array is measured at the anode. b. 260-hour lifetesting results for two cathode chips in the whole-chip test fixture. The constant field applied to each was $-5.7 \mathrm{~V} / \mu \mathrm{m}$. Levels of emitted current varied widely from chip to chip, but cathodes capable of producing over $10 \mu \mathrm{A}$ over hundreds of hours were common. (At constant accelerating voltage, the mini-EPMA will produce the same X-ray line ratios regardless of current level. However, current level will affect the time needed to achieve good $\mathrm{S} / \mathrm{N}$ in the X-ray measurement.) Post-test examination via SEM revealed no obvious erosion or other signs of degradation of the CNT forests. Cathodes have not yet been tested for more than 260 hours.

\section{CONCLUSIONS}

The development of new instrument technology is inherently interdisciplinary. A deep knowledge of current technological advancements, intersected with the near- to mid-term mission needs driven by the planetary science landscape, are needed to best target the most compelling opportunities for new instruments to have an impact on future mission capabilities. The two examples here build upon state-of-the-art techniques in planetary instrumentation, mass spectrometry and X-ray spectroscopy, to address the next challenges defined by outstanding scientific questions in planetary composition and evolution.

\section{REFERENCES}

[1] Freissinet, C., Glavin, D. P., Mahaffy, P. R., Miller, K. E., Eigenbrode, J. L., Summons, R. E., Brunner, A. E., Buch, A., Szopa, C., Archer, P. D., Franz, H. B., Atreya, S. K., Brinckerhoff, W. B., Cabane, M., Coll, P., Conrad, P. G., Des Marais, D. J., Dworkin, J. P., Fairén, A. G., François, P., Grotzinger, J. P., Kashyap, S., Kate, I. L., Leshin, L. A., Malespin, C. A., Martin, M. G., Martin-Torres, F. J., McAdam, A. C., Ming, D. W., Navarro-González, R., Pavlov, A. A., Prats, B. D., Squyres, S. W., Steele, A., Stern, J. C., Sumner, D. Y., Sutter, B., Zorzano, M.-P., and the MSL Science Team ( 2015), Organic molecules in the Sheepbed Mudstone, Gale Crater, Mars. J. Geophys. Res. Planets, 120, 495- 514. Doi: 10.1002/2014JE004737. 
[2] Jennifer L. Eigenbrode, Roger E. Summons, Andrew Steele, Caroline Freissinet, Maëva Millan, Rafael NavarroGonzález, Brad Sutter, Amy C. McAdam, Heather B. Franz, Daniel P. Glavin, Paul D. Archer Jr., Paul R. Mahaffy, Pamela G. Conrad, Joel A. Hurowitz, John P. Grotzinger, Sanjeev Gupta, Doug W. Ming, Dawn Y. Sumner, Cyril Szopa, Charles Malespin, Arnaud Buch, Patrice Coll (2018), Organic matter preserved in 3-billion-year-old mudstones at Gale crater, Mars, Science, 08 June 2018: 1096-1101.

[3] C. C. Porco, P. Helfenstein, P. C. Thomas, A. P. Ingersoll, J. Wisdom, R. West, G. Neukum, T. Denk, R. Wagner, T. Roatsch, S. Kieffer, E. Turtle, A. McEwen, T. V. Johnson, J. Rathbun, J. Veverka, D. Wilson, J. Perry, J. Spitale, A. Brahic, J. A. Burns, A. D. DelGenio, L. Dones, C. D. Murray, S. Squyres (2006), Cassini Observes the Active South Pole of Enceladus, Science, 311, 1393-1401.

[4] Amanda R. Hendrix, Terry A. Hurford, Laura M. Barge, Michael T. Bland, Jeff S. Bowman, William Brinckerhoff, Bonnie J. Buratti, Morgan L. Cable, Julie Castillo-Rogez, Geoffrey C. Collins, Serina Diniega, Christopher R. German, Alexander G. Hayes, Tori Hoehler, Sona Hosseini, Carly J.A. Howett, Alfred S. McEwen, Catherine D. Neish, Marc Neveu, Tom A. Nordheim, G. Wesley Patterson, D. Alex Patthoff, Cynthia Phillips, Alyssa Rhoden, Britney E. Schmidt, Kelsi N. Singer, Jason M. Soderblom, and Steven D. Vance (2019), The NASA Roadmap to Ocean Worlds, Astrobiology, 19, https://doi.org/10.1089/ast.2018.1955

[5] Discovery Program Announcement 2019, https://discovery.larc.nasa.gov/

[6] NF-4 Press Release: NASA Invests in Concept Development for Missions to Comet, Saturn Moon Titan; https://www.nasa.gov/press-release/nasa-invests-in-concept-development-for-missions-to-comet-saturn-moon-titan

[7] CLPS Press Release: NASA Announces New Partnerships for Commercial Lunar Payload Delivery Services; https://www.nasa.gov/press-release/nasa-announces-new-partnerships-for-commercial-lunar-payload-deliveryservices

[8] M. G. G. T. Taylor, N. Altobelli, B. J. Buratti, and M. Choukroun (2015), The Rosetta mission orbiter science overview: the comet phase, Philosophical Transactions of the Royal Society A: Mathematical, Physical and Engineering Sciences, 375, http://doi.org/10.1098/rsta.2016.0262

[9] Monica M. Grady, Ian P. Wright, Cécile Engrand, Sandra Siljeström (2018), The Rosetta Mission and the Chemistry of Organic Species in Comet 67P/Churyumov-Gerasimenko, Elements, 14, 95-100.

[10] E. Beshore et al., "The OSIRIS-REx asteroid sample return mission," 2015 IEEE Aerospace Conference, Big Sky, MT, 2015,pp.1-14. doi: 10.1109/AERO.2015.7118989.

[11] Xiang Li, Ryan M. Danell, William B. Brinckerhoff, Veronica T. Pinnick, Friso van Amerom, Ricardo D. ArevaloJr., Stephanie A. Getty, Paul R. Mahaffy, Harald Steininger, and Fred Goesmann (2015), Detection of Trace Organics in Mars Analog Samples Containing Perchlorate by Laser Desorption/Ionization Mass Spectrometry, Astrobiology 15:2, 104-110.

[12] S. A. Getty et al., "Molecular analyzer for Complex Refractory Organic-rich Surfaces (MACROS)," 2017 IEEE Aerospace Conference, Big Sky, MT, 2017, pp. 1-11.; doi: 10.1109/AERO.2017.7943706

[13] S. A. Getty et al., "Organics Analyzer for Sampling Icy Surfaces: A liquid chromatograph-mass spectrometer for future in situ small body missions," 2013 IEEE Aerospace Conference, Big Sky, MT, 2013, pp. 1-8.; doi: 10.1109/AERO.2013.6497391

[14] Jessica S. Creamer, Maria F. Mora, and Peter A. Willis (2016), Enhanced Resolution of Chiral Amino Acids with Capillary Electrophoresis for Biosignature Detection in Extraterrestrial Samples, Anal. Chem., 89 (2), pp 1329-1337

[15] Cornish, T. J. and Cotter, R. J. (1993), A curved-field reflectron for improved energy focusing of product ions in time-of-flight mass spectrometry. Rapid Commun. Mass Spectrom., 7: 1037-1040. doi:10.1002/rcm.1290071114

[16] Tanaka, K.; Waki, H.; Ido, Y.; Akita, S.; Yoshida, Y.; Yoshida, T.; Matsuo, T. (1988). "Protein and Polymer Analyses up to m/z 100000 by Laser Ionization Time-of flight Mass Spectrometry". Rapid Communications in Mass Spectrometry 2 (20): 151-3. doi:10.1002/rcm.1290020802

[17] Bastin, G. F. and H. J. M. Heijligers (1990). Quantitative electron probe microanalysis of ultralight elements (boron-oxygen). Scanning 12, 225-236. 
[18] Nash, W. P. (1992). Analysis of oxygen with the electron microprobe: Applications to hydrated glass and minerals. American Mineralogist 77, 453-457.

[19] McGee, J. J. and K. Keil (2001). Application of electron probe microanalysis to the study of geological and planetary materials. Microscopy and Microanalysis 7, 200-210.

[20] Bunch, T. E., J. H. Wittke, and R. L. Korotev (2006, September). Petrology and Composition of Lunar Feldspathic Breccias NWA 2995, Dhofar 1180 and Dhofar 1428. Meteoritics and Planetary Science, Vol. 41, Supplement, Proceedings of $69^{\text {th }}$ Annual Meeting of the Meteoritical Society, held August 6-11, 2006 in Zurich, Switzerland., \#5254.

[21] Hurt, S. M., A. E. Rubin, and J. T. Wasson (2012, June). Fractionated matrix composition in CV3 Vigarano and alteration processes on the CV parent asteroid. Meteoritics and Planetary Science 47, 1035-1048.

[22] Allwood, A., B. Clark, D. Flannery, J. Hurowitz, L. Wade, T. Elam, M. Foote, and E. Knowles (2015). Texturespecific elemental analysis of rocks and soils with PIXL: The Planetary Instrument for X-ray Lithochemistry on Mars 2020. In Aerospace Conference, 2015 IEEE, pp. 1-13. IEEE.

[23] Prigozhin, G., K. Gendreau, J. P. Doty, R. Foster, R. Remillard, A. Malonis, B. LaMarr, M. Vezie, M. Egan, J. Villasenor, Z. Arzoumanian, W. Baumgartner, F. Scholze, C. Laubis, M. Krumrey, and A. Huber (2016, July). NICER instrument detector subsystem: Description and performance. SPIE 9905, 99051I.

[24] Sise et al. (2009), Journal of Electron Spectroscopy and Related Phenomena 175, 76-86

[25] Radauscher, E. J., A. D. Keil, M. Wells, J. J. Amsden, J. R. Piascik, C. B. Parker, B. R. Stoner, and J. T. Glass (2015, November). Chemical Ionization Mass Spectrometry Using Carbon Nanotube Field Emission Electron Sources. Journal of The American Society for Mass Spectrometry 26, 1903-1910. 